\title{
The prevalence and antimicrobial responses of Shigella Isolates in HIV-1 infected and uninfected adult diarrhoea patients in north west Ethiopia
}

\author{
${ }^{1}$ Berhanu Andualem, ${ }^{1}$ Afeworke Kassu, ${ }^{1}$ Ermias Diro, ${ }^{1}$ Feleke Moges, ${ }^{1}$ Molla Gedefaw
}

\begin{abstract}
Background: Shigella is one of the diarrhoea causing organisms found in HIV positive patients. But so far, the pattern of diarrhoeal agents caused by Shigella in AIDS patients has not been determined

Objective: This study is thus aimed at determining the prevalence, antimicrobial susceptibility and resistance of Shigella isolates in HIV positive subjects.

Methods: All stool samples taken from the subjects of this study were plated on the MacConkey agar and incubated at $35-37^{\circ} \mathrm{C}$ for 24 or $48 \mathrm{hrs}$. Biochemical and antimicrobial sensitivity testing were carried out by using the standard methods.

Result: Out of the 391 subjects included in the study, 199(63.8\%) HIV seropositive and 113 seronegative patients had acute and chronic diarrhoea while 79 were HIV seropositive without diarrhoea. Of the 27 (8.7) Shigella isolates taken from the diarrhea patients, 11 (3.5\%) were from HIV positive subjects. All Shigella isolates were found to be sensitive against norfloxacin (100\%), gentamicin (97\%), polymyxin B (97\%) and kanamycin (93\%). The most frequent resistance observed was to chloramphenicol (62\%), tetracycline (86\%) and ampicillin (100\%). The frequency of resistance of Amp, Sex, Ch, TTc was found to be very high when compared with other patterns of resistance.

Conclusion: The high proportion of HIV seropositive patients who had diarrhea in the absence of identified Shigella strains strongly indicates the existence of other diarrhoeagenic agents or mechanisms. Detailed investigation is important to get comprehensive information for better treatment of diarrhoea in HIV /AIDS patients. According to this finding, norfloxacin, gentamicin, polymyxin B, kanamycin and nalidixic acid might be used as drugs of choice for empirical treatment. On the other hand, amplicilin, tetracycline and chloramphenicol may not be used as the drugs of choice for the treatment of Shigella infection unless culture and sensitivity tests are done prior to treatment. [Ethiop.J.Health Dev. 2006;20(2):99-105]
\end{abstract}

\section{Introduction}

The HIV/AIDS pandemic that started over twenty years ago is still extremely dynamic and expanding worldwide. At present 39.4 million people are estimated to be living with HIV/AIDS in the world, of which, over $64 \%$ are living in sub-Saharan Africa. HIV is now the leading cause of death worldwide in the age group of 15-24 (1). In Ethiopia, the first HIV infections were identified in 1984, and the first AIDS cases were reported in 1986. Currently Ethiopia ranks third among the most heavily HIV/AIDS affected countries (India and South Africa) in the world. According to the current estimate, close to three million people are infected with HIV. According to the UNAIDS 2004 report, the HIV prevalence rate in Ethiopia is about $4.4 \%$ (2). The health impact of HIV/AIDS is associated with lowering the immune status which makes it easily vulnerable for infectious diseases.

Diarrhoea is formally defined as an increase in daily stool weight above 200g. Typically, the patient may also describe an abnormal increase in stool liquidity and frequency (3). Diarrhoea is considered acute when it lasts for less than 14 days and chronic when more than two weeks (3).

A number of infectious diseases (including candidiasis, tuberculosis, and CNS mass lesions) are known to be common in HIV patients in Ethiopia (4), and diarrhoea is one of them. It is one of the clinical manifestations of HIV infection in both developing and developed countries in all seasons. Moreover, chronic diarrhoea associated with weight loss is often common in HIV-1 infected individuals (5). Among those etiological agents of diarrhoea, the most important infective agents are of bacterial, viral, parasitic and protozoal origin.

Acquired immunodeficiency syndrome (AIDS) caused by HIV infection predisposes individuals to several diarrhoeagenic bacterial diseases of various species like Salmonella, Shigella, Yersinia, Campylobacter, Vibrio cholerae and diarrhoeagenic E. coli (6) in addition to several parasitic, viral and protozoal aetiological agents (7).

Despite the determination of the prevalence of diarrhoea caused by parasites (7) the pattern of diarrhoea agents caused by diarrhoeagenic Shigella in AIDS patients has not been determined. This study, therefore, attempts to determine the prevalence of various species like Shigella agents in AIDS patients with diarrhoea.

\section{Methods}

Study design and subjects: A cross-sectional study was conducted in Gondar College of Medicine and Health

\footnotetext{
${ }^{1}$ Department of App. Biology, App. Natural Sciences Faculty, University of Gondar, P.O. Box 196, Gondar, Ethiopia
} 
Sciences Hospital (GCMHS). The study subjects were in and out patients with diarrhoea and who visited GCMHS between March 29, 2003 and October 5, 2004. The sample size for the study was calculated by considering a 95\% level of confidence, $90 \%$ proportion of studies done in Addis Ababa (7) and Jimma (8) respectively, with a $5 \%$ margin and a $20 \%$ contingency. A total of 391 subjects were included in the study based on information about the frequency of visits of such patients to GCMHS. Out of these, 312 were HIV seropositive and negative patients with acute and chronic diarrhoea while 79 were HIV seropositives without diarrhoea (control group). Data were collected by using a pre-structured and pretested questionnaire. The variables include sociodemographic characteristics of the patient associated risk factors, clinical history as well as physical findings and laboratory data.

Physicians collected demographic data and $5 \mathrm{ml}$ of venous blood were taken by laboratory technicians from each subject after informed consent and appropriate pretest counseling by a counselor. The serum was then collected in sterile test tubes, was labeled, and was transported to the main laboratory as soon as possible. To assure patient confidentiality, code numbers were used to identify patient data and serum samples. Names of patients were not recorded in the questionnaire.

In the laboratory, the serum was separated and kept in Nunc tubes at $-20^{\circ} \mathrm{C}$. The presence of HIV antibodies was confirmed by using Determine Neg (Abbott Laboratories, USA), Capilus (Trintibiotech, USA) and Uni-Gold $^{\mathrm{TM}}$ (Trintibiotech, USA) according to the standard screening guidelines set by WHO (9).

Diarrheic stool specimens were also collected fresh in sterile containers. The physical nature of diarrhoea was examined and processed in the GCMHS laboratory immediately after collection.

Isolation of stool pathogens: The collected specimens were plated on the MacConkey agar after inoculation using a sterile loop. All the inoculated plates on the McConkey agar were incubated at $35-37^{0} \mathrm{C}$ for $24 \mathrm{hrs}$.

The MacConkey (BBL) plates were examined for nonlactose fermenting colonies. By using a sterile straight wire, a single colony of either type from the plates was picked and inoculated in about $3 \mathrm{ml}$ of nutrient broth. This was incubated at $35-37^{\circ} \mathrm{C}$ for $2-4$ hours until growth was ascertained by turbidity. The suspension was then tested biochemically. In brief, identification of Gramnegative bacteria was performed with the help of biochemical tests which routinely included triple sugar iron (TSI) agar slant, lysine iron (LI) agar slant, urea agar slant, Simmon`s citrate agar slant, SIM medium, mannitol broth (1\%), glucose broth (1\%), oxidase reagents and hydrogen peroxide (BBL) (10). The reference strain for shigella (NBL SC 530) was used for control purposes throughout the study.

Antimicrobial sensitivity testing: Susceptibility testing of all strains was done on the Muller-Hinton agar with commercial antibiotic discs using the single disc diffusion technique of Bauer et al., (11) against

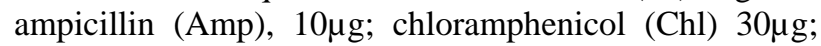

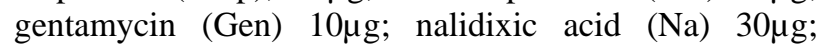

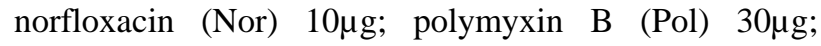

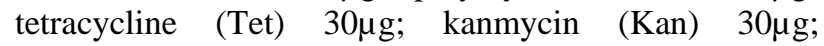

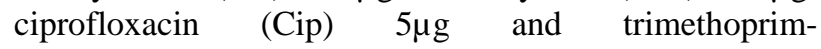

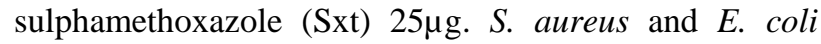
strains susceptible to all the antibiotics tested were used as controls.

Finally, the diameters of inhibition zones were measured in millimeters using a caliper. The interpretation of the measurement as sensitive, intermediate and resistant was made according to a standard zone size interpretive chart (12). The intermediate readings were considered as sensitive for the assessment of the data.

Statistical analysis was performed using SPSS. The data were entered and analyzed using SPSS Version 10 statistical program.

\section{Results}

Sample collection and laboratory processing was carried out for 19 months during the study period. A total of 391 subjects were examined to determine the prevalence of Shigella isolates. Out of these, 312 were HIV seropositive and negative subjects with acute and chronic diarrhoea (case study), while 79 were HIV seropositive without diarrhoea (control group).

In diarrhoea patients within the age group of 15-24 years the proportion of males to females in HIV seropositive subjects was $3.5 \%$ and $4.5 \%$ respectively. Similarly in diarrhoea patients within the 25-34 year age category the proportion of males to females was $15.7 \%$ and $17.0 \%$. It was observed that females in the age category of 15-34 years were affected more than males. Within the age groups of more than 35 years the proportion of HIV seropositive males was greater than the females (Table 1).

With regard to marital status $(\mathrm{P}<0.01)$ and occupational distribution $(\mathrm{P}<0.01)$ the prevalence of HIV in diarrhoea patients has shown significant variation in the study subjects (Table2).

The history of herpes zoster in HIV seropositive and negative diarrhoea, patients was observed. Among the herpes zoster - positive diarroea patients $(n=58), 47 / 58$ 
Table 1: Age distribution of HIV seropositive and negative diarrhoea patients visiting GCMHS hospital, 2003/4

\begin{tabular}{|c|c|c|c|c|c|}
\hline \multirow{2}{*}{\multicolumn{2}{|c|}{$\begin{array}{c}\text { Age } \\
\text { (year) }\end{array}$}} & \multicolumn{3}{|c|}{ Case study, N = 312} & \multirow{2}{*}{$\begin{array}{l}\text { Control group, } \\
N=79 \text { (\%) }\end{array}$} \\
\hline & & HIV+ (\%) & HIV- (\%) & Total (\%) & \\
\hline \multirow[t]{2}{*}{$15-24$} & Male & $11(3.5)$ & $40(12.8)$ & $51(6.3)$ & $6(7.6)$ \\
\hline & Female & $14(4.5)$ & $10(4.1)$ & $23(7.4)$ & $11(13.9)$ \\
\hline \multirow[t]{2}{*}{$25-34$} & Male & $49(15.7)$ & $16(5.1)$ & $65(20.8)$ & $12(15.2)$ \\
\hline & Female & $53(17.0)$ & $17(5.4)$ & $70(22.4)$ & $23(29.1)$ \\
\hline \multirow[t]{2}{*}{$35-44$} & Male & $34(10.9)$ & $7(2.2)$ & $41(13.1)$ & $12(15.2)$ \\
\hline & Female & $21(6.7)$ & $9(2.9)$ & $30(9.60$ & $6(7.6)$ \\
\hline \multirow[t]{2}{*}{$>45$} & Male & $12(3.8)$ & $9(2.9)$ & $21(6.7)$ & $4(5.1)$ \\
\hline & Female & $5(1.6)$ & $5(1.6)$ & $11(3.5)$ & 5 96.3) \\
\hline \multirow[t]{3}{*}{ Total } & Male & $106(34.0)$ & $74(23.7)$ & $180(57.7)$ & $33(41.8)$ \\
\hline & Female & $93(29.8)$ & $39(12.5)$ & $132(42.3)$ & $46(58.2)$ \\
\hline & Total & $199(63.8)$ & $113(36.2)$ & $312(100)$ * & $79(100)$ \\
\hline
\end{tabular}

Table 2: Marital status and occupation distribution of HIV seropositive and negative diarrhoea patients visiting GCMHS hospital, 2003/4

\begin{tabular}{|c|c|c|c|c|}
\hline \multirow{2}{*}{$\begin{array}{l}\text { Marital Status (A) and } \\
\text { Occupation distribution (B) }\end{array}$} & \multicolumn{3}{|c|}{ Case study N = 312} & \multirow{2}{*}{$\begin{array}{l}\text { Control } \\
\text { group } \\
N=79(\%)\end{array}$} \\
\hline & HIV+ (\%) & HIV- (\%) & Total (\%) & \\
\hline \multirow[t]{8}{*}{ A) } & 99 (31.7) & $57(18.3)$ & $156(50)$ & $38(48.1)$ \\
\hline & 49.7 & 50.4 & 50 & 48.1 \\
\hline & $56(17.9)$ & $49(15.4)$ & $105(33.7)$ & $22(27.8)$ \\
\hline & 28.2 & 43.4 & 33.7 & 27.8 \\
\hline & $39(12.5)$ & $4(1.3)$ & $43(13.8)$ & $19(24.1)$ \\
\hline & 19.6 & 3.5 & 13.8 & 24.1 \\
\hline & $5(1.6)$ & $3(1.0)$ & $8(2.6)$ & - \\
\hline & 2.5 & 2.7 & 2.6 & - \\
\hline $\begin{array}{l}\text { Total } \\
\text { Government }\end{array}$ & $199(63.8)$ & $113(36.2)$ & $312(100)$ * & 79 (100) \\
\hline employee & $43(13.8)$ & $23(7.4)$ & $66(21.2)$ & $15(19.0)$ \\
\hline \% with HIV & 21.6 & 20.4 & 21.2 & 19.0 \\
\hline Farmer & $31(9.9)$ & $22(7.1)$ & $53(17.0)$ & $6(7.6)$ \\
\hline \% with HIV & 15.6 & $19.5 \%$ & 17.0 & 7.6 \\
\hline Student & $12(3.8)$ & $36(11.5)$ & $48(15.4)$ & $7(8.9)$ \\
\hline \% with HIV & 6.0 & 31.9 & 15.4 & 8.9 \\
\hline Housewife & $30(9.6)$ & $12(3.8)$ & $42(13.5)$ & $16(20.3)$ \\
\hline \% with HIV & 15.1 & 10.6 & 13.5 & 20.3 \\
\hline Others & 31 (9.9) & $22(7.1)$ & 53 (17.0) & 35 (44.3) \\
\hline \% with HIV & 15.6 & 19.5 & 17.0 & 44.3 \\
\hline Total & $199(63.8)$ & $113(36.2)$ & $312(100)$ * * & 79 (100) \\
\hline
\end{tabular}

* $\mathrm{P}<0.01 \quad$ ** $\mathrm{P}<0.01$

(81.0\%) were HIV positive and 11/58 (19.0\%) were HIV seronegative $(\mathrm{P}<0.002)$.

Among the 199 HIV infected diarrhoea patients, 79/199 (39.7\%) and 120/199 (60.3\%) had acute and chronic diarrhoea respectively $(\mathrm{P}<0.001)$ (Table 3). The prevalence of chronic diarrhoea was higher than that of acute diarrhoea. The association of parasites $(n=99)$ with diarrhoea type (acute and chronic diarrhoea) is shown on Table 3. Intestinal parasites were detected $59 / 99$ (59.6\%) in patients with chronic diarrhea and in 40/99 (40.4\%) of patients with acute diarrhoea.

The prevalence of Shigella isolates in HIV positive and negative subjects is shown in Table 4. Shigella was isolated from both HIV positive and negative diarrhoea 
patients. Of the 27 (8.7) Shigella isolates taken from diarrhea patients, 11 (3.5\%) were from HIV positive subjects while 16 (5.1\%) were from the HIV negative ones. On the other hand, out of the HIV positive nondiarrhoea patients, 2 (2.5\%) of the 79 patients were Shigella positive. In this study, the nature of the diarrhoea as well as fever and appetite were not found to be statistically associated with Shigella isolates in diarrhoea patients. On the other hand, previous episodes of such dirrhoea and weight loss were associated with Shigella isolates $(\mathrm{P}<0.025$ and 0.027), (Table 4).

\begin{tabular}{|c|c|c|c|c|}
\hline \multirow{2}{*}{$\begin{array}{l}\text { A) Type of diarrhoea } \\
\text { in association with } \\
\text { HIV }\end{array}$} & \multicolumn{3}{|c|}{ Case study N = 312} & \\
\hline & HIV+ (\%) & HIV- (\%) & Total (\%) & \\
\hline Acute diarrhoea & $79(25.3)$ & $66(21.2)$ & 145 (46.5) & \\
\hline With HIV & 39.7 & 58.4 & 46.5 & \\
\hline Chronic diarrhoea & $120(38.5)$ & $47(15.1)$ & 167 (53.5) & \\
\hline With HIV & 60.3 & 41.6 & 53.5 & \\
\hline Total & $199(63.8)$ & $113(36.2)$ & $312(100)^{*}$ & \\
\hline $\begin{array}{l}\text { B) Type of } \\
\text { diarrhea in association } \\
\text { with parasites }\end{array}$ & $\begin{array}{l}\text { Parasites } \\
\text { Positive }\end{array}$ & $\begin{array}{l}\text { Parasites } \\
\text { Negative }\end{array}$ & Total & \\
\hline Acute & $40(12.8)$ & 105 (33.7) & 145 (46.5) & \\
\hline$\%$ With parasite & 40.4 & 49.3 & 46.5 & \\
\hline Chronic & $59(18.9)$ & $108(34.6)$ & 167 (53.5) & \\
\hline \%Within parasite & 59.6 & 50.7 & 53.5 & \\
\hline Total & $99(31.7)$ & $213(68.3 \%)$ & $312(100)$ & \\
\hline \multirow[t]{2}{*}{ Shigella isolates } & \multicolumn{3}{|c|}{ Case study $\mathrm{N}=312$} & Control \\
\hline & $\mathrm{HIV}+$ & HIV - & tal & $\mathrm{N}=79$ \\
\hline Positive & $11(3.5)$ & $16(5.1)$ & $27(8.6)$ & $2(2.5)$ \\
\hline Negative & $188(60.3)$ & $97(31.1)$ & $285(91.4)$ & 77 (97.5) \\
\hline Total & 199 (63..8) & $113(36.2)$ & $312(100)$ ** & $79(100)$ \\
\hline
\end{tabular}

* $=\mathrm{P}<0.012 \quad$ ** $=\mathrm{P}<0.009$

Table 4: Diarrhoea status of Shigella isolates

\begin{tabular}{|c|c|c|c|c|}
\hline \multirow[t]{2}{*}{ Clinical symptoms } & & \multicolumn{3}{|c|}{ Shigella isolates } \\
\hline & & Positive (\%) & Negative (\%) & Total (\%) \\
\hline \multirow[t]{4}{*}{ Nature of diarrhoea } & Watery & $9(2.9)$ & $129(41.3)$ & $138(44.2)$ \\
\hline & Bloody & $13(4.2)$ & $82(26.3)$ & $95(30.4)$ \\
\hline & Mucoid & $5(1.6)$ & 74 (23.7) & 79 (25.3) \\
\hline & Total & $27(8.7)$ & $285(91.3)$ & $312(100)$ \\
\hline \multirow[t]{3}{*}{ Abdominal cramp } & Yes & $20(6.4)$ & $212(67.9)$ & $232(74.4)$ \\
\hline & No & $7(2.2)$ & $73(23.4)$ & $80(25.6)$ \\
\hline & Total & $27(8.7)$ & $285(91.3)$ & $312(100)$ \\
\hline \multirow[t]{3}{*}{ Previous episode } & Yes & $9(2.9)$ & $163(52.2)$ & $172(55.1)$ \\
\hline & No & $18(5.8)$ & $122(39.1)$ & $140(44.9)$ \\
\hline & Total & $27(8.7)$ & 285 (91.3) & $312(100)$ * \\
\hline \multirow[t]{3}{*}{ Weight loss } & Yes & $8(2.9)$ & $149(45.8)$ & 172 (50.3) \\
\hline & No & $19(6.1)$ & $136(43.6)$ & $155(49.7)$ \\
\hline & Total & $27(8.7)$ & $285(91.3)$ & $312(100)$ ** \\
\hline \multirow[t]{3}{*}{ Fever } & Yes & $18(5.8)$ & $138(44.2)$ & $156(50.0)$ \\
\hline & No & $9(2.9)$ & $147(47.1)$ & $156(50.0)$ \\
\hline & Total & $27(8.7)$ & $285(91.3)$ & $312(100)$ \\
\hline \multirow[t]{3}{*}{ Appetite } & Yes & $6(1.9)$ & $67(21.5)$ & $73(23.4)$ \\
\hline & No & $21(6.7)$ & $218(69.9)$ & $239(76.6)$ \\
\hline & Total & $27(8.7)$ & $285(91.3)$ & $312(100)$ \\
\hline
\end{tabular}




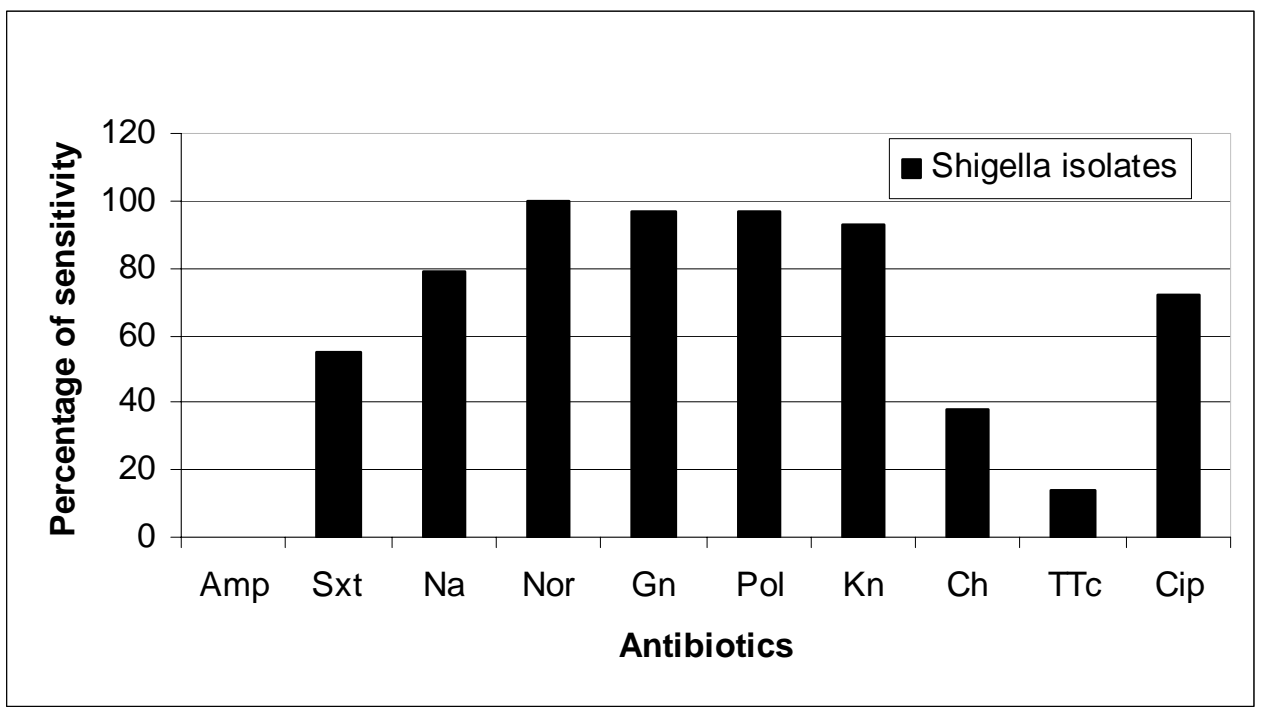

Figure 1: Sensitivity pattern of Shigella isolates against certain antibiotics

$$
\begin{aligned}
\text { Amp } & =\text { Ampicillin } \\
\mathrm{Na} & =\text { Nalidixic acid } \\
\mathrm{Gn} & =\text { Gentamycin } \\
\mathrm{Kn} & =\text { Kanamycin } \\
\mathrm{TTc} & =\text { Tetracycline }
\end{aligned}
$$

$$
\begin{aligned}
& \text { Sxt }=\text { Trimethoprim-sulphamethoxazole } \\
& \text { Nor }=\text { Norfloxacin } \\
& \text { Pol }=\text { Poymyxin B } \\
& \text { Ch }=\text { Chloramphenicol } \\
& \text { Cip }=\text { Ciprofloxacin }
\end{aligned}
$$

The antimicrobial sensitivity tests of Shigella isolates against 10 antimicrobial agents are shown in Figure 1. All the strains were found to be sensitive against norfloxacin (100\%), while 97\%, 97\%, and 93\% of Shigella strains were found to be sensitive against gentamicin, poymyxin $\mathrm{B}$ and kanamycin, respectively. Moreover, $79 \%$, and $72 \%$ of Shigella strains were sensitive against nalidixic acid and ciprofloxacin, respectively. About 55\% of strains were sensitive against trimethoprim-sulphamethoxazole. The most frequent resistance observed was to chloramphenicol (62\%), tetracycline (86\%) and ampicillin (100\%).

Resistance to one or more drugs in Shigella isolates was observed for $29(100 \%)$ of the isolates. Out of the 29 Shigella strains, 28 (96.6\%) were multiple resistant. In all, fourteen different patterns of resistance were noted. The frequency of resistance for Amp, Sxt, Ch, TTc, (i.e.) is the highest in comparison with other patterns of resistance.

\section{Discussion}

The result of this study in terms of HIV infection agrees with that indicated on the 2005 UNAIDS report (2). It was observed that the highest infection rates are concentrated in the 15-34 year age group. Within this age group, prevalence among females was found to be greater than that of males. Other than gender, marital status and occupation have also shown impact on the distribution of HIV. The association of Herpes zoster infection and HIV was also found to be high (81\%).
In this investigation, chronic diarrhoea was found to be more common than acute diarrhoea in HIV positive subjects. On the other hand, acute diarrhoea was more common than chronic diarrhoea in HIV negative subjects.

The $8.7 \%$ frequency of isolation of Shigella in this study was lower than the isolated $(9.0 \%)$ strains reported by Mogessie (13) and the $11.7 \%$ isolation rate reported by Asrat et al, (14) at Tikur Anbessa and Ethio-Swedish Childrens' Hospitals and the $11.6 \%$ isolation rate of stool of diarrhoea patients Stoll et al, (15) from Bangladesh.

The prevalence rate of Shigella isolates (8.7\%) in this work was almost $7.1 \%$ of Shigella strains isolated by Meche et. al, (16) in Addis Ababa between January and July, 1995 and the 7\% isolation rate reported by Ai et al., (17) from south Vietnam but much higher than the $5.8 \%$ frequency of isolation in Addis Ababa (18) and 1.2\% isolation rate from Malaysia (19).

In this investigation of diarrhoea patients, the prevalence of Shigella isolates in HIV positive cases was 3.5\% and 5.1\% were from HIV negative subjects. From this study one can possibly conclude that Shigella strains did not seem to be the main aetiological enteric pathogenic agents when compared with other diarrhoeagenic agents. So the high proportion of HIV seropositive patients who had diarrhoea in the absence of identified Shigella strains strongly indicates the existence of other diarrhoeagenic agents or mechanisms. Detailed investigation is 
important to get comprehensive information for the better treatment of diarrhoea in HIV /AIDS patients.

As opposed to the $100 \%$ sensitivity of trimethoprimsulphamethoxazole that has been reported by Mogessie (13), the frequency of sensitivity of Shigella strains against trimethoprim-sulphamethoxazole was $55 \%$. The frequency of sensitivity to all the drugs observed in this study (64.5\%) was higher than the $16.7 \%$ and $30 \%$ reported by Mogessie (13) Afeworki and Yetnebersh (20), respectively. According to Zeleke's (21) report, about $50 \% 100 \%$, and $100 \%$ of the isolated Shigella strains were sensitive against tetracycline, trimethoprimsulphamethoxazoe and chloramphenicol, respectively, while $14 \%, 55 \%$ and $38 \%$ of Shigella isolates of this study were sensitive against tetracycline, trimethoprimsulphamethoxazole and chloramphenicol, respectively. The high rate of occurrence of resistance to these antimicrobial drugs may be due to the extensive use of these drugs for the treatment of diarrhoeal diseases. From this observation, one can conclude that there is increased emergence of resistant strains against ampicillin. Such a trend was also reported by various investigators (22-24).

This study shows that of all the drugs tested, a single drug resistance was found against ampicillin in $3.4 \%$ of the Shigella strains, which was different from the report of the single drug resistance against chloramphenicol, sulphadiazene, tetracycline, streptomycin or cephlothin reported by Mogessie (13) and carbenicillin by Andualem and Geyid (25). The frequency of ampicillin resistance $(100 \%)$ in this investigation was much higher (75\%) than that reported by Andualem and Geyid, (25), (52.3\%), Mogessie (13) and (22\%) by Afeworki and Yetnebersh (20).

The emergence of resistant Shigella strains against the commonly used antimicrobial agents is being reported in Ethiopia. This indicates the need for newer and more effective drugs of choice for the treatment of shigellosis (26). According to this study, norfloxcin, gentamicin polymyxin $\mathrm{B}$, kanamycin and nalidixic acid may be drugs of choice for the treatment of shigellosis, particularly in regions where there is high prevalence of multiple drug resistant strains since their sensitivity pattern is $>79 \%$.

The occurrence of resistant strains in this and other previous studies may be the result of the widespread use of these drugs. This may cause selective pressure of resistance problems on the enteric bacteria as a whole circulating in the community (27).

According to this finding, norfloxacin, gentamicin, polymyxin $\mathrm{B}$, kanamyin and nalidixic acid may be used as drugs of choice for empirical treatment. On the other hand, amplicilin, tetracycline and chloramphenicol may not be used as drugs of choice for the treatment of
Shigella infection unless culture and sensitivity tests are done prior to treatment.

In underdeveloped countries like Ethiopia, besides limiting therapeutic options and the high cost of alternative effective agents, resistant organisms may lead to longer hospitalization and an increased risk of death. The development of new antimicrobial agents may offer short-term solution to this problem but in the long run more effective measures such as health education and further research on the prevention of infections through quality sanitation, vaccination and immunization should be emphasized.

\section{Acknowledgment}

The author would like to thank the University of Gondar Presidents office and the research and Publication Office for their facilities and financial support to the experimental work. I also like to extend my gratitude to Ato Mulugeta Aemero for his assistance in reviewing the manuscript.

\section{References}

1. UNAIDS. 2004 Report on the Global HIV/AIDS Epidemic: $4^{\text {th }}$ Global Report.

2. UNAIDS. 2005 Report on the Global HIV/AIDS Epidemic: $5^{\text {th }}$ Global Report.

3. Harrison TR, Braunwald E and Fauci. Harrison's principles of internal medicine. USA 1998; 1: 237.

4. Daniel F. HIV associated opportunistic infections in Ethiopia. Faculty of Medicine, Addis Ababa University, WWW. Gap-a org, 2004.

5. Colebunders R. Persistent diarrhoea in Zairian AIDS patients: an endoscopic and histologic study. Gut.1990;26: 687-1691.

6. WHO. Manual for the disease control programs: Control of diarrhoea disease. WHO/CDD/SER/81.5 Rev. 1984; 1:3-5

7. Fisseha B, Petros B, Woldemichael $\mathrm{T}$ and Mohammed H. Diarrhoea-associated parasitic infectious agents in AIDS patients within Addis Ababa Hospitals. Ethiop J Health Dev. 1999;13:163173.

8. Awole M, Gegre_Selassie S, Kassa T and Kibtu G. Prevalence of intestinal parasites in HIV-infected adult parasites in southwestern Ethiopia. Ethiop J Health Dev. 2003;17(1):71-78.

9. WHO and UNAIDS. Revised recommendations for the selection and use of HIV antibody tests. WHO Wkly Epidemiol. 1997;72:81-87.

10. Power DA, McCuen PJ. Manual of BBL products and laboratory products: Becton Dickinson Microbiology systems. Elsevier, New York, USA 1988;6:336-337.

11. Bauer AW, Kirby WMM, Sherris JC and Turck M. Antibiotic susceptibility testing by a standardized single disc method. AMER J Clin Pathol. 1966;36:493-496.497. 
12. Matsen JM and Barry AL. Susceptibility testing: diffusion test procedures. In: Manual of Clinical Microbiology. $2^{\text {nd }}$ ed. American Society for Microbiology Washington D.C., USA. 1974;421.

13. Mogessie A. The prevalence of Salmonella, Shigella and Yersinia entrocolitica in adult diarrhoea outpatients in some hospitals in Addis Ababa. MSc thesis Addis Ababa University, AA. Ethiopia. 1983.

14. Daniel A, Hathaway A. and Ekwall E. Studies on enteric Campaylobacterosis on Tikur Anbessa and Ethio-Swedish Children's Hospitals, Addis Ababa, Ethiopia. Ethiop Med J 1999;37:71-83.

15. Stoll BJ, Glass RI, Huq MI, Kuan MV, Bann H. and Holt J. Epidemiologic and clinical features of patients infected with Shigella who attached a diarrhoea disease hospital in Bangladesh. J Infect Dis. 1982;88:177-83.

16. Mache A, Mengistu Y and Cowly S. Shigella serogroups identified from adult diarrhoea outpatients in Addis Ababa, Ethiopia: Antibiotic resistance and plasmid profile analysis. E Afr Med J 1997;74(3):179-82.

17. Ai NV, Hanh, ND,Van LT and Ho TM. Bacteriological and epidemiological studies on Shigella infection in South Vietnam. Bul Soc Path Exot 1975;68(3):262-6.

18. Andualem B and Geyid A. The prevalence of Yersinia entrocolitica isolates in comparison to those of the commonly encountered enteropathogens causing diarrhoea among Ethiopian patients in Addis Ababa. Ethiop Med J, 2003;41:257-266.
19. Kan SK and Chan MK. Annual and geographical distribution of Shigella serotypes in Sabah, Malaysia from 1974 to 1978. Med J Malaysia 1980;35(1):913.

20. Afeworki $G$ and Yetneberh L. Multiple drug resistance within Shigella serogroups. Ethiop. Med. J. 1980;18:7-14.

21. Zeleke WT. Survey of gastroenteritis causing bacterial pathogens in feeding bottle contents in Addis Ababa. MSc. Thesis, Addis Ababa University. 1990.

22. Byers PA, Dupont, HL and Golscidt MC. Antimicrobial susceptibility of Shigella isolated in Houton. Texas, in 1974. Antimicro Agents Chemother. 1976;9:288-291.

23. Ngu VV. Observations in Shigella isolates in the Bach Mai hospital (Hanoi) in the course of 3 years (1974-1976). Santo Publ. 1977;20:461-464.

24. Stool BJ. Glass RI, Huq M.I, Khan MV, Bann, H and Holt J. Epidemiology and clinical features of patients infected with Shigella who attended a diarrhea disease hospital in Bangladesh. J Infect Dis. 1982;146:177-183.

25. Andualem B and Geyid A. Antimicrobial responses of Yersinia entrocolitica isolates in comparison to other commonly encountered bacteria that cause diarrhoea. E Afr Med J, 2003;80(8):13-17. 21.

26. Messale $G$ and Alebachew T. Shigella species fro Addis Ababa: Frequency of isolation and in vitro drug sensitivity. J Hyg Camb. 1982;88:47-55.

27. Bennett JV. Antibiotic use in animals and humans salmonellosis. J Infect Dis. 1980;142:631-633. 